\title{
Multimodal Analysis of Reaction Pathways of Cathode Materials for Lithium Ion Batteries
}

Sooyeon Hwang ${ }^{1}$, Xiao $\mathrm{Ji}^{2}$, Seong-Min Bak ${ }^{1}$, Jianming Bai ${ }^{1}$, Ke Sun ${ }^{1}$, Xiulin Fan ${ }^{2}$, Hong Gan ${ }^{1}$, Chunsheng Wang ${ }^{2}$ and Dong $\mathrm{Su}^{1}$

${ }^{1}$ Brookhaven National Laboratory, Upton, New York, United States, ${ }^{2}$ University of Maryland, College Park, Maryland, United States

Conversion mechanism in lithium ion batteries provides higher capacity than intercalation mechanism since multiple numbers of electrons and lithium ions are associated. However, poor cycling stability, large voltage hysteresis, and low energy efficiency have been great challenges of conversion reaction. To address those issues, conversion-type electrode materials have been reformed via doping or substituting other elements. For example, iron fluorides $\left(\mathrm{FeF}_{2}, \mathrm{FeF}_{3}\right)$ have modified as iron oxyfluorides $\left(\mathrm{FeF}_{1-\mathrm{x}} \mathrm{O}_{\mathrm{x}}\right)$, showing enhanced long-term stability. ${ }^{[1][2]}$ Furthermore, co-substituted (both anion and cation substituted) $\mathrm{Fe}_{0.9} \mathrm{Co} 0.1 \mathrm{OF}$ (FeCoOF) was demonstrated excellent cycling stability. (capacity of $350 \mathrm{mAh}$ $\mathrm{g}^{-1}$ at a current of $500 \mathrm{~mA} \mathrm{g-}^{1}$ for 1000 cycles ${ }^{[3]}$ Substituting anion and cation in iron fluoride has been suggested as an effective method to achieve better reversibility but understanding of lithiation reactions in co-substituted FeCoOF is not clear. This work takes advantage of ex-situ/ in-situ synchrotron X-ray based techniques and transmission electron microscopy to elucidate structural changes with lithium ion, which may provide fundamental insights into modifying conversion-type materials.

Figure 1 presents discharge-charge curves and pair distribution function patterns acquired at each potential. As lithium ions were inserted, structural changes were noticed both at short-range and longrange. However, long-range ordering was nearly maintained even at $1 \mathrm{~V}$, indicating absence of conversion reaction. Figure 2 shows lithiation induced structural evolution of a single FeCoOF nanorod observed in real time. As lithiation proceeds, the width of nanorods shows a stepwise increase, particularly A in figure $2 \mathrm{~d}$, which may indicate multiple steps of lithiation occur. Considering that conversion reaction takes place around $2 \mathrm{~V}_{\text {in }} \mathrm{FeF}_{3},{ }^{[4]}$ co-substitution $\mathrm{Co}$ and $\mathrm{O}$ into iron fluoride may change thermodynamic features of lithiation reactions by lowering the initiation potential for conversion reaction. Instead, phase transformations occur at long-range order, which may help maintaining structural integrity during operation, eventually, achieving cycling stability [5]. 

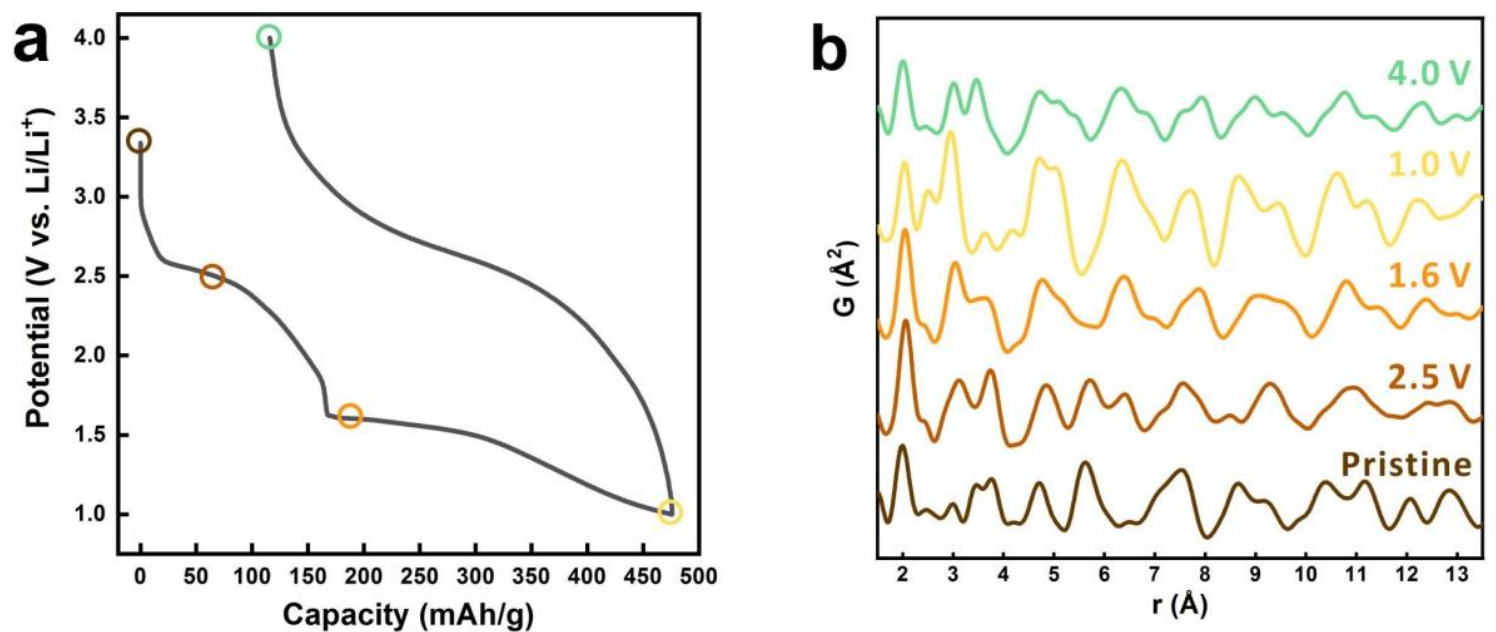

Figure 1. (a) discharge-charge profile of FeCoOF at a current density of $50 \mathrm{mAg}-1$. (b) PDF patterns acquired at different potentials.
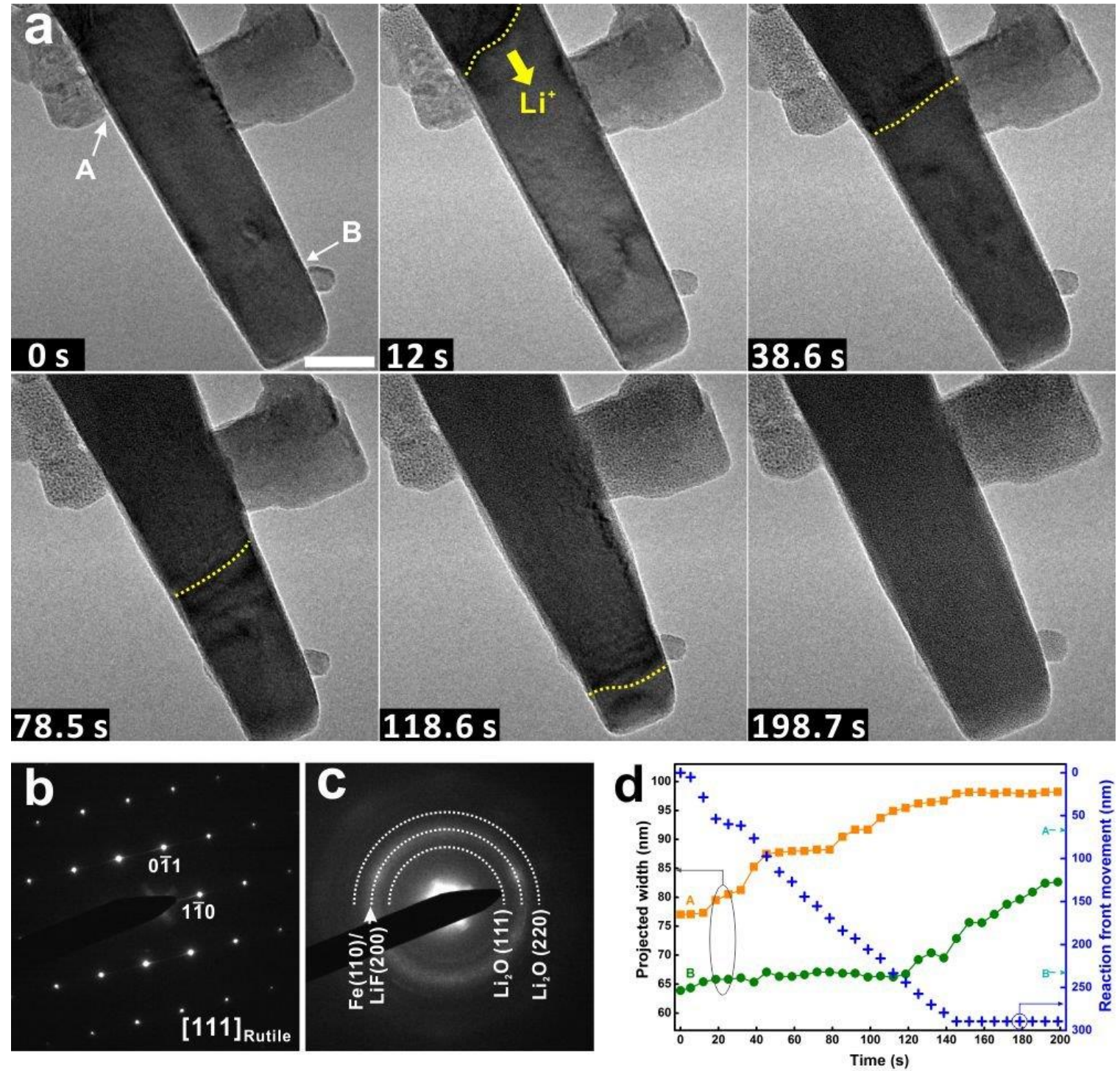
Figure 2. A time-series of bright-field TEM images during lithiating a single nanorod of FeCoOF. Electron diffraction patterns acquired (b) before and (c) after in situ lithiation. (d) Projected width and reaction front movement in function of lithiation time. Widths of nanowire are tracked at two different positions of $\mathrm{A}$ and $\mathrm{B}$ shown in (a).

References

[1] S.-W. Kim, N. Pereira, N. A. Chernova, F. Omenya, P. Gao, M. S. Whittingham, G. G. Amatucci, D. Su, F. Wang, ACS Nano 2015, 9, 10076-10084.

[2] M. Sina, K.-W. Nam, D. Su, N. Pereira, X. Q. Yang, G. G. Amatucci, F. Cosandey, J. Mater. Chem. A2013, 1, 11629-11640.

[3] X. Fan, E. Hu, X. Ji, Y. Zhu, F. Han, S. Hwang, J. Liu, S. Bak, Z. Ma, T. Gao, et al., Nat. Commun. 2018, 9, 2324.

[4] F. Badway, F. Cosandey, N. Pereira, G. G. Amatucci, J. Electrochem. Soc. 2003, 150, A1318A1327.

[5] This work is supported by the Center for Functional Nanomaterials, which is a US DOE Office of Science Facility, at Brookhaven National Laboratory under Contract No. DE- SC0012704. 